\title{
Do Regulatory Bioequivalence Requirements Adequately Reflect the Therapeutic Equivalence of Modified-Release Drug Products?
}

\author{
Laszlo Endrenyi $^{1}$ and Laszlo Tothfalusi ${ }^{2}$ \\ ${ }^{1}$ Department of Pharmacology and Toxicology, University of Toronto, Toronto, ON, Canada; ${ }^{2}$ Semmelweis University, \\ Department of Pharmacodynamics, Budapest, Hungary
}

Received, March 16, 2010; Revised, May 7, 2010; Accepted, May 7, 2010; Published, May 8, 2010.

\begin{abstract}
Purpose. To demonstrate that current regulatory requirements for bioequivalence (BE) do not always reflect therapeutic equivalence. To investigate the potential usefulness of an additional metric, the partial AUC. Methods. Pharmacokinetic information was reviewed and evaluated on the pharmacokinetics of modified-release methylphenidate and nifedipine products. Results. In studies of modified-release products of methylphenidate as well as of nifedipine, traditional regulatory criteria found two formulations to be bioequivalent even though their concentration profiles strongly diverged during the period of absorption. An additional metric, partial AUC, discriminated strongly between the concentrations of the drug products. Conclusions. The current regulatory criteria for the acceptance of BE do not always reflect the therapeutic equivalence of modified-release drug products. With some modified-release products, the application of an additional metric, the partial AUC, yields an improved discriminatory representation.
\end{abstract}

\section{INTRODUCTION}

The complicated pharmacokinetic (PK) characteristics of some modified-release (MR) products have become the focus of recent discussions (1) including a meeting of the FDA Advisory Committee for Pharmaceutical Science and Clinical Pharmacology $(2,3)$. The pattern of drug release from these formulations is tailored to give rise to preset pharmacodynamic (PD) responses. It is, therefore, important that second-entry drug products demonstrate PK patterns sufficiently similar to those of the reference product. Strong deviations between the PK patterns of some products will be illustrated when traditional regulatory criteria actually indicate their bioequivalence.

\section{Regulatory requirements for the bioequivalence of modified-release formulations}

The current regulatory expectation of the U.S. Food and Drug Administration (FDA) for declaring the bioequivalence of two drug products is based on the 90\% confidence limits around the ratio of geometric (GMR) means of the area under the plasma drug concentration-time curve (AUC) as well as that of the values of the maximum plasma drug concentrations $\left(\mathrm{C}_{\max }\right)$ that must be within 0.80 to 1.25 (4). The requirement of Health Canada is the same for AUC while for $\mathrm{C}_{\max }$ only the point estimate of GMR is expected to be between 0.80 and $1.25(5,6)$.

\section{COMPARISONS OF COMPLICATED CONCENTRATION PROFILES OF MR FORMULATIONS}

\section{Example 1: Concentration profiles and therapeutic features of methylphenidate formulations}

Methylphenidate is a mild central nervous system stimulant used in the treatment of attention deficit hyperactivity disorder. The administration of the drug, however, is associated with acute tolerance (i.e., reduced efficacy) that manifests itself in approximately $4 \mathrm{~h}$ (7). Conventional modifiedrelease formulations of the drug that are shown to be effective for up to $8 \mathrm{~h}$ (8) have been developed with the intention of the convenience of daily dosing. Subsequent modified-release formulations

Corresponding Author: Dr. Laszlo Endrenyi, Department of Pharmacology and Toxicology University of Toronto, Toronto, ON, Canada M5S 1A8. l.endrenyi@utoronto.ca 
of methylphenidate have been developed that maintain their efficacy for extended periods (9).

Such products included Metadate CD (UCB S.A., Brussels, Belgium) and Concerta (Ortho-Janssen, Toronto, ON, in Canada); the latter maintained its therapeutic effect for $12 \mathrm{~h}$.

The Metadate CD capsules contain 30\% immediate-release and 70\% extended-release beads. The resulting concentration profile is biphasic after oral administration. The active ingredient of Concerta tablets are divided into $22 \%$ in the outer layer for immediate-release and the remaining 78\% incorporated in the core for an osmotic-controlled release oral delivery system (OROS). The resulting concentration-time profile of this product is multiphasic. Following the oral administration of a single tablet, the methylphenidate concentration rises rapidly, typically, within about one hour. An approximate plateau is maintained for about 4 hours after which the concentration ascends further, at a moderate rate, until a peak is reached in about 6 to 8 hours. Thereafter the concentration declines at a comparatively gentle rate. The difference between Metadate CD and Concerta is that the former gives rise to a substantially higher initial concentration and an ascending phase without the plateau period observed with the latter (Figure 1). Consequently, the peak concentration is attained sooner with Metadate CD as compared with Concerta.

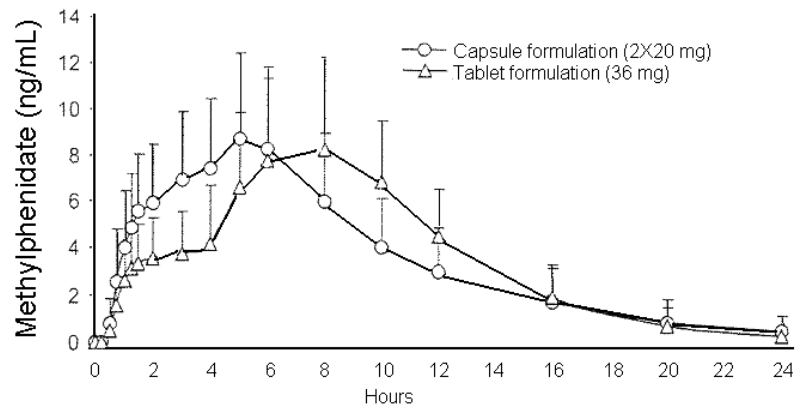

Figure 1. Pharmacokinetic patterns of Metadate CD (capsule) and Concerta (tablet). From reference 10 with permission.

Such pharmacokinetic characteristics which are achieved by careful design of the formulations (11) have been reported to successfully overcome the diminishing effect of the acute tolerance. The immediate release portion provides a quick response and the ascending phase compensates for the loss of efficacy secondary to the development of acute tolerance. (e.g., 12, 13).

It is generally expected that test and reference formulations in studies of bioequivalence should have closely similar clinical properties, i.e., that their safety and efficacy features should be highly comparable. Pharmacokinetic comparability (bioequivalence) with pharmaceutical equivalents is usually assumed to serve as a convenient surrogate for therapeutic equivalence, i.e., clinical similarity. However, the currently used metrics do not always reflect therapeutic equivalence. For example, it is possible that two preparations show similar AUC and $\mathrm{C}_{\max }$ values that appear to satisfy the regulatory requirements for bioequivalence without consideration given to the conspicuous difference between their concentration profiles. This was the case in the comparison of the Concerta and Metadate CD extended-release preparations (Figure 1; ref. 10). Their relevant pharmacokinetic parameters [AUC(0-tlast) and $\mathrm{C}_{\max }$ ] are similar and satisfy the criteria for bioequivalence (Table 1). Nevertheless, their concentration profiles are different and show substantial deviations in the early stages of drug release.

The methylphenidate example illustrates that satisfying the current bioequivalence criteria between two drug products does not necessarily ensure the similarity in the drug release characteristics. For methylphenidate the therapeutic and/or adverse consequences are related to the drug concentration profile, hence, bioequivalence based on the current two metrics does not guarantee therapeutic equivalence. This is clearly the case in comparisons of Concerta with Metadate CD and with several other extended-release methylphenidate preparations.

\section{Example 2: Concentration profiles of nifedipine formulations}

Anschütz et al. (2010) recently compared the time courses and pharmacokinetic characteristics of two modified-release nifedipine formulations (14). Both drug products used OROS formulations. The reference product was the $60 \mathrm{mg}$ Adalat XL tablet (Bayer Healthcare AG, Leverkusen, Germany) with a bilayer pump system, while the test formulation 
Table 1. Bioequivalence metrics comparing Metadate CD and Concerta (from reference 10)

\begin{tabular}{|c|c|c|c|}
\hline & GMR (\%) & \multicolumn{2}{|c|}{ 90\% Confid. Limits } \\
\hline \multicolumn{4}{|c|}{20 mg Metadate capsule vs. 18 mg Concerta tablet } \\
\hline AUC(0-tlast) & 107.78 & 103.74 & 111.97 \\
\hline $\mathrm{C}_{\max }$ & 99.39 & 93.82 & 105.29 \\
\hline AUC(0-4hr) & 69.92 & 66.24 & 73.82 \\
\hline AUC(0-6hr) & 77.94 & 74.21 & 81.85 \\
\hline \multicolumn{4}{|c|}{ 2x20 mg Metadate capsules vs. 36 mg Concerta tablet } \\
\hline AUC(0-tlast) & 113.44 & 108.93 & 118.14 \\
\hline $\mathrm{C}_{\max }$ & 111.28 & 103.36 & 119.80 \\
\hline AUC(0-4hr) & 63.80 & 60.15 & 67.68 \\
\hline AUC(0-6hr) & 72.15 & 68.01 & 76.54 \\
\hline \multicolumn{4}{|c|}{ 3x20 mg Metadate capsules vs. 54 mg Concerta tablet } \\
\hline AUC(0-tlast) & 110.08 & 105.70 & 114.83 \\
\hline $\mathrm{C}_{\max }$ & 101.05 & 93.64 & 109.04 \\
\hline AUC(0-4hr) & 65.50 & 61.75 & 69.48 \\
\hline AUC(0-6hr) & 73.24 & 69.03 & 77.69 \\
\hline
\end{tabular}

was a $60 \mathrm{mg}$ Gen-Nifedipine extended-release tablet with a monolayer pump [Mylan Pharmaceuticals ULC (previously Genpharm ULC), Etobicoke, ON, Canada].

Figure 2 illustrates the time courses of average concentrations after the administration of a single dose to 26 fasted subjects (14). Both the peak concentrations and AUCs of the two formulations appear to be comparable. However, the shapes of the concentration profiles are quite different. The test product has a longer lag-time and a later $\mathrm{T}_{\max }$ than the reference formulation. Importantly, the plateau phase is shorter for the test than the reference product; the half-value durations were calculated to be 25.2 and $32.7 \mathrm{~h}$, respectively.

Table 2 (modified from Table 5 of reference 14) illustrates that the current bioequivalence metrics (AUC and $\mathrm{C}_{\max }$ ) of the two drug products were indeed comparable. However, this would not be confirmed by the differing concentration profiles of the two formulations. The separation of the two concentration profiles is demonstrated by the strongly differing partial AUCs which were recorded to 9 hours following drug administration. 


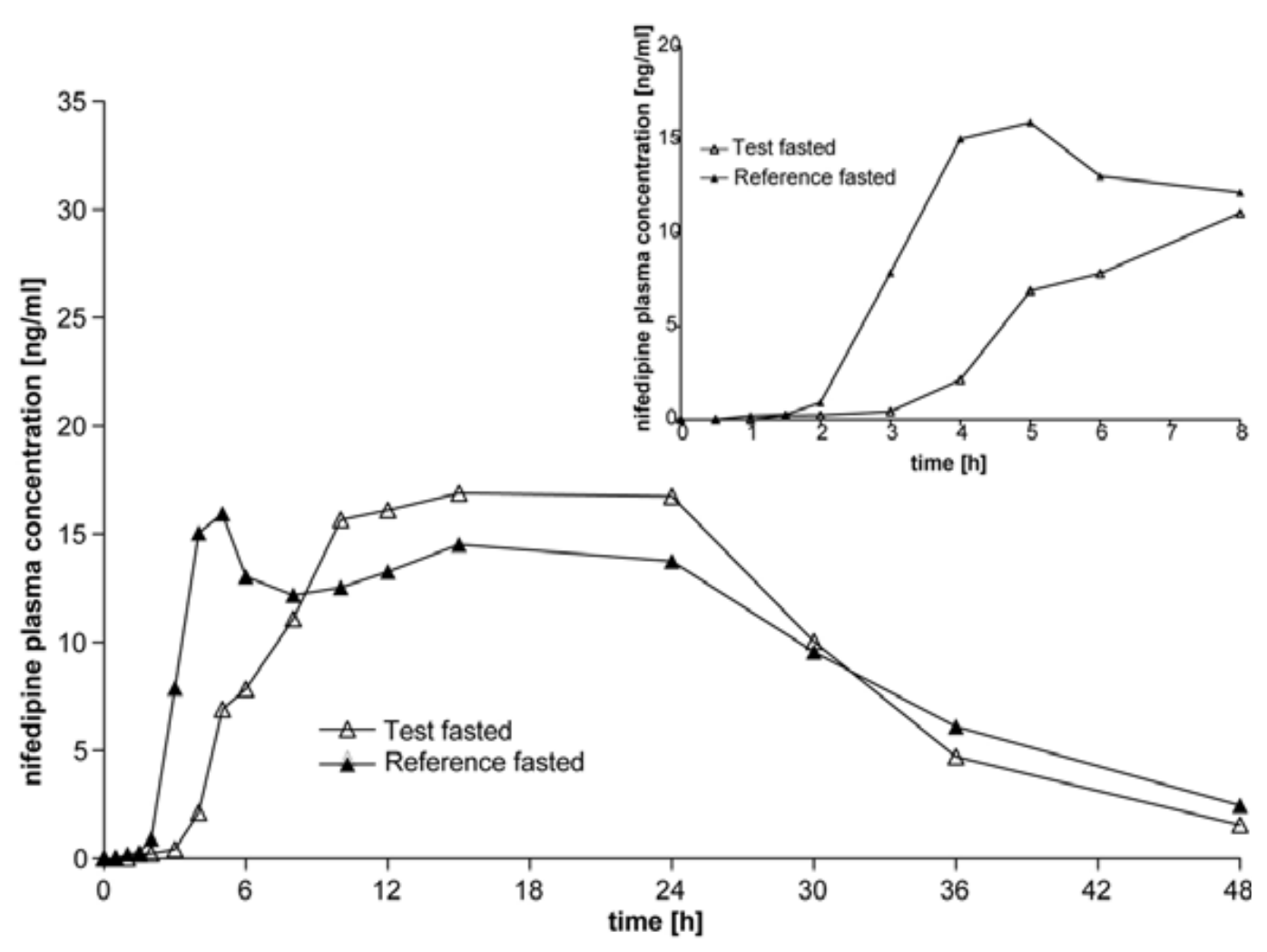

Figure 2. Pharmacokinetic patterns of nifedipine following administration of the reference and test products. The insert depicts the first 8 h post-dose. From reference 14 with the permission.

Table 2. Bioequivalence parameters comparing Gen-Nifedipine ER and Adalat XL Adopted from reference 14.

\begin{tabular}{lccc}
\hline & GMR (\%) & \multicolumn{2}{c}{ 90\% Confidence Limits } \\
\hline AUC(0-tlast) & 91.8 & 79.9 & 105.5 \\
C $_{\max }$ & 99.8 & 88.6 & 112.4
\end{tabular}

modified-release drug products. This has been recently discussed at a joint meeting of AAPS and FIP (1) and also, more recently, at an FDA advisory committee meeting $(2,3)$. Davit (2, 15) noted that "multiphasic $\begin{array}{lllll}\text { AUC(0-9 h) } & \mathbf{5 4 . 8} & \mathbf{4 5 . 8} & \mathbf{6 5 . 5} & \text { modified-release drug products present issues }\end{array}$ for bioequivalence evaluation that are not

GMR: Ratio of geometric means (Test/Reference) encountered with simple modified-release products”. Midha and McKay $(16,17)$ stated

\section{DISCUSSION}

More reliable characterization of the absorption phase of some modified-release products using partial AUCs

The two examples provided herein illustrate that the currently applied two bioequivalence parameters (AUC and $\mathrm{C}_{\max }$ ) are insufficient to characterize comparisons involving various that "for some newer CR/MR drug products with different drug release mechanisms, the present regulatory recommendations may not be adequate”.

For assessing bioequivalence, ideally, the concentration profiles of the two drug products should be compared (e.g. 18). In practice, however, it would be more feasible to consider additional metrics at least for 
some multiphasic modified-release formulations, notably, partial AUC (pAUC) as a measure for the onset of therapeutic and/or adverse responses, and as an index of early exposure (19-21). pAUC is an AUC determined between given time points. For an onset response, pAUC is typically evaluated from the time of drug administration (usually, $\mathrm{t}=0$ ) until a predetermined time-point.

The metric of pAUC has found increasing regulatory recognition in recent years. The general guidance of FDA for bioavailability and bioequivalence studies for orally administered drugs (4) recommends the use of pAUC as an early exposure measure "on the basis of appropriate clinical efficacy/safety trials and/or pharmacokinetic/pharmacodynamic studies that call for better control of drug absorption into the systemic circulation".

One of the reasons why $\mathrm{C}_{\max }$ is a preferred bioequivalence metric is because it is generally assumed that the peak concentration is directly related to the safety or the maximum effect of a drug. The underlying PK/PD relationship behind this assumption is the simple $\mathrm{E}_{\max }$ model which often does not hold. For example, partial AUC can be a better predictor of safety than $\mathrm{C}_{\max }$ when acute tolerance develops to adverse effects (22).

A recent FDA draft guidance on the bioequivalence of extended-release tablets of zolpidem tartrate (Ambien CR ${ }^{\circledR}$ ) recommends that $90 \%$ confidence intervals for the ratio of geometric means be established not only for AUC and $\mathrm{C}_{\max }$ but also for pAUCs from 0 to $1.5 \mathrm{~h}$ and from 1.5 $\mathrm{h}$ to the last measurable time point (23). Moreover, FDA is considering the application of partial AUC, as an additional metric, to the evaluation of $\mathrm{BE}$ of modified-release methylphenidate preparations (2). In this case, the determination of pAUC from 0 to 3 $\mathrm{h}$ and from $3 \mathrm{~h}$ to the last measurable concentration is envisaged in fasted studies, and from 0 to $4 \mathrm{~h}$ and from $4 \mathrm{~h}$ to last measurable time point in fed investigations (2).

\section{Application of pAUC to determine bioequivalence of the modified-release methylphenidate and nifedipine products}

As depicted in Table 1, AUC values $0-4$ and 0-6 h discriminate well between the two modified-release methylphenidate formulations at three dose levels; the ratios of the geometric means are between 60 and $75 \%$, and the upper $90 \%$ confidence limit is not higher than $82 \%$. It is also noteworthy that the confidence limits of pAUCs are narrow, thereby indicating fairly small withinsubject variations. The discriminating power of pAUC is also apparent for nifedipine products (Table 2). The ratio of geometric means is 0.548 , and its $90 \%$ confidence limits are from 0.458 to 0.655 . Consequently, pAUC clearly signals the differences in the concentration-time profiles of the two drug products.

Based on the available data, in
considering pAUC as a powerful
discriminating index in bioequivalence
assessment, it is difficult to choose a fixed
time interval to fit all drug products. Instead,
the timing is best decided based on the
therapeutic relevance of the drug
concentration-time pattern of the specific
product.

\section{CONCLUSIONS}

The current criteria for assessing bioequivalence of some modified-release products with different concentration-time profiles are inadequate. This may result in declaring bioequivalence of products that are not therapeutically equivalent. Partial AUC appears to be a powerful discriminating index and its inclusion as an additional criterion will assure therapeutic parity of two products. The time interval for partial AUC should be decided based on the therapeutic relevance of the concentration-time profile of the product. 


\section{REFERENCES}

1. Chen, M-L, Shah, VP, Ganes, D, et al. Challenges and opportunities in establishing scientific and regulatory standars for assuring therapeutic equivalence of modified-release products: Workshop summary report. Eur. J. Pharm. Sci. 2010; 40: 148-153.

2. Davit, BM. Use of partial AUC: case studies and BE approaches. Meeting of the FDA Advisory Committee for Pharmaceutical Science and Clinical Pharmacology, Silver Spring, MD, April 13, 2010. http://www.fda.gov/downloads/AdvisoryCom mittees/CommitteesMeetingMaterials/Drugs/

AdvisoryCommitteeforPharmaceuticalScience andClinicalPharmacology/UCM209320.pdf (accessed: 5/4/2010)

3. Use of partial area under the curve (AUC) for the evaluation of abbreviated new drug applications (ANDAs) for products with complex pharmacokinetic profiles. Background Information for the FDA Meeting of the Advisory Committee for Pharmaceutical Science and Clinical Pharmacology, FDA/CDER, March 2010. http://www.fda.gov/downloads/AdvisoryCom mittees/CommitteesMeetingMaterials/Drugs/ AdvisoryCommitteeforPharmaceuticalScience andClinicalPharmacology/UCM207955.pdf (accessed: 5/4/2010).

4. FDA. Bioavailability and Bioequivalence Studies for Orally Administered Drug Products-General Considerations, Guidance for Industry. Rockville, MD: Food and Drug Administration, Center for Drug Evaluation and Research (CDER), March, 2003.

5. Health Canada. Guidance for Industry. Conduct and Analysis of Bioavailability and Bioequivalence Studies. Part A: Oral Dosage Formulations Used for Systemic Effects. Health Canada, Ottawa, ON,1992.

6. Health Canada. Guidance for Industry. Conduct and Analysis of Bioavailability and Bioequivalence Studies. Part B: Oral Modified Release Formulations. Health Canada, Ottawa, ON, 1996.

7. Srinivas, NR, Hubbard, JW, Quinn, D, and Midha, KK. Enantioselective pharmacokinetics and pharmacodynamics of dl-threo-methylphenidate in children with attention deficit hyperactivity disorder. Clin. Pharmacol. Ther. 1992; 52: 561-568.
8. Pelham WE, Sturges J, Hoza J, et al. Sustained release and standard methylphenidate effects on cognition and social behavior in children with attention deficit disorder. Pediatrics, 1987; 80: 491501.

9. Pelham WE, Gnagy EM, Burrows-Maclean L, et al. Once-a-day Concerta methylphenidate versus three-times-daily methylphenidate in laboratory and natural settings. Pediatrics, 2001; 107: 1417-1418 (Abstract e105).

10. Gonzalez, MA, Pentikis, HS, Anderl, N, et al. Methylphenidate bioavailability from two extended release formulations. Int. J. Clin. Pharmacol. Ther. 2002; 40: 175-184.

11. Swanson, JM, Gupta, S, Williams, I, et al. Efficacy of a new pattern of delivery of methylphenidate for the treatment of ADHD activity level in the classroom and on the playground. J. Amer. Acad. Child Adolesc. Psychiatry, 2002; 41: 1306-1314.

12. Lopez, F, Silva, R, Pestreich, L, and Muniz, R. Comparative efficacy of two once daily methylphenidate formulations (Ritalin LA and Concerta) and placebo in children with attention deficit hyperactivity disorder across the school day. Paediatric Drugs, 2003; 5: 545-555.

13. Greenhill, LL, DeCroy, HH, Hirshey-Dirksen, SJ, and Hatch, SJ. Comparisons of classroom deportment in 6-12 year olds with ADHD after administration of two once-daily extended release methylphenidate (MPH) formulations. Amer. Psychiatric Assoc., San Francisco, CA, May 21, 2003.

14. Anschütz, M., Wonnemann, M., Schug, B., Toal, C., Donath, F., Pontius, A.,Pauli, K., Brendel, E., and Blume, H. Differences in bioavailability between $60 \mathrm{mg}$ of nifedipine osmotic push-pull systems after fasting and fed administrations. Int. J. Clin. Pharmacol. Ther. 2010; 48: 158-170.

15. Davit, BM. Special considerations for establishing bioequivalence of MR products. AAPS workshop: "Challenges and Opportunities in Establishing Scientific and Regulatory Standards for Assuring 
Therapeutic Equivalence of Modified-Release Products”, Baltimore, MD, October 1-2, 2009.

16. Midha, KK and McKay, G. Bioequivalence of MR products: $P K$ and therapeutic equivalence. AAPS workshop: "Challenges and Opportunities in Establishing Scientific and Regulatory Standards for Assuring Therapeutic Equivalence of Modified-Release Products”, Baltimore, MD, October 1-2, 2009.

17. Midha, KK and McKay, G. Use of partial area under the curve for BE assessment of products with complex PK profiles; a view point. Meeting of the FDA Advisory Committee for Pharmaceutical Science and Clinical Pharmacology, Silver Spring, MD, April 13, 2010. http://www.fda.gov/downloads/AdvisoryCom mittees/CommitteesMeetingMaterials/Drugs/ AdvisoryCommitteeforPharmaceuticalScience andClinicalPharmacology/UCM209320.pdf. (accessed: 5/4/2010).

18. Pereira, LM. Bioequivalence testing by statistical shape analysis. J. Pharmacokin. Pharmacodyn. 2007; 34: 451-484.
19. Chen, ML. An alternative approach for assessment of rate of absorption in bioequivalence studies. Pharm. Res. 1992; 9: 1380-1385.

20. Chen, ML, Lesko, L, and Williams, RL Measures of exposure versus measures of rate and extent of absorption. 2001; Clin. Pharmacokin. 2001; 40: 565-572.

21. Endrenyi, L, Csizmadia, F, Tothfalusi, L, Balch, AH, and Chen, ML. The duration of measuring partial AUC's for the assessment of bioequivalence. Pharm. Res. 1998; 15: 399-404.

22. Tothfalusi, L, Speidl, S, and Endrenyi, L. Exposure-response analysis reveals that clinically important toxicity difference can exist between bioequivalent carbamazepine tablets. Br. J. Clin. Pharmacol. 2008; 65: 110-122.

23. FDA. Draft Guidance on Zolpidem. Food and Drug Administration, Center for Drug Evaluation and Research (CDER) Rockville, MD, August, 2009. http://www.fda.gov/Drugs/GuidanceComplian ceRegulatoryInformation/Guidances/ucm0813 39 (accessed: 5/4/2010) 